\title{
A FRAMEWORK FOR MIMETIC DISCRETIZATION OF THE ROTATING SHALLOW-WATER EQUATIONS ON ARBITRARY POLYGONAL GRIDS*
}

\author{
J. THUBURN ${ }^{\dagger}$ AND C. J. COTTER ${ }^{\ddagger}$
}

\begin{abstract}
Accurate simulation of atmospheric flow in weather and climate prediction models requires the discretization of the governing equations to have a number of desirable properties. Although these properties can be achieved relatively straightforwardly on a latitude-longitude grid, they are much more challenging on the various quasi-uniform spherical grids that are now under consideration. A recently developed scheme - called TRiSK - has these desirable properties on grids that have an orthogonal dual. The present work extends the TRiSK scheme into a more general framework suitable for grids that have a nonorthogonal dual, such as the equiangular cubed sphere. We also show that this framework fits within the wider framework of mimetic discretizations and discrete exterior calculus. One key ingredient is the definition of certain mapping operators that are discrete analogues of the Hodge star operator, enabling the definition of a compatible inner product. Discrete Coriolis terms are also included within the mimetic framework, and in such a way as to conserve energy and ensure that discrete geostrophic balance can be maintained; this requires the definition of a further mapping operator, with special properties, that transfers the discrete velocity field from the primal to the dual grid.
\end{abstract}

Key words. Coriolis term, discrete exterior calculus, geostrophic balance, Hodge star, nonorthogonal mesh

AMS subject classifications. 65M06, 65M08, 76U05

DOI. $10.1137 / 110850293$

1. Introduction. Developers of global weather and climate models, for both operational prediction and research use, are exploring a variety of quasi-uniform alternatives to the traditional latitude-longitude horizontal gridding of the sphere; the latitude-longitude grid suffers from resolution clustering at the poles, and this is expected to lead to poor scaling on the massively parallel computers that will be needed for very high resolution simulation. Atmospheric flow is strongly multiscale in both space and time. Consequently, the accuracy of simulation depends on the discretization satisfying a number of desirable properties - see section 1.1. These properties can be achieved relatively straightforwardly on a latitude-longitude grid. Obtaining them on alternative grids is a significant challenge, and that is the topic of this paper.

The shallow-water equations describe the motion of a layer of fluid on a twodimensional surface in a rotating reference frame. Here we use them in their so-called "vector invariant" form:

$$
\begin{aligned}
\frac{\partial \phi}{\partial t}+\nabla \cdot(\mathbf{v} \phi) & =0 \\
\frac{\partial \mathbf{v}}{\partial t}+\zeta \mathbf{v}^{\perp}+\nabla\left(\phi+\frac{1}{2}|\mathbf{v}|^{2}\right) & =\mathbf{0} .
\end{aligned}
$$

*Submitted to the journal's Computational Methods in Science and Engineering section October 4, 2011; accepted for publication (in revised form) February 10, 2012; published electronically May 1, 2012. This work was supported by the UK Natural Environment Research Council.

http://www.siam.org/journals/sisc/34-3/85029.html

${ }^{\dagger}$ College of Engineering, Mathematics and Physical Sciences, University of Exeter, Exeter, EX4 4QF, UK (j.thuburn@exter.ac.uk).

${ }^{\ddagger}$ Department of Aeronautics, Imperial College, London, SW7 2AZ, UK (colin.cotter@imperial. ac.uk). 
Here, $\phi$ is the geopotential (proportional to the fluid depth), $\mathbf{v}$ is the horizontal velocity, $\mathbf{v}^{\perp}=\mathbf{k} \times \mathbf{v}$ is the velocity rotated through a right angle, where $\mathbf{k}$ is the unit vertical vector, and $\zeta=f+\xi$ is the absolute vorticity, where $f$ is the Coriolis parameter associated with the rotation of the reference frame and $\xi=\mathbf{k} \cdot \nabla \times \mathbf{v}=-\nabla \cdot \mathbf{v}^{\perp}$ is the relative vorticity. This form of the equations is valid both in planar geometry and on curved manifolds such as a sphere, provided $\nabla$ and $\nabla \cdot()$ are interpreted as the appropriate covariant operators. In general $f$ may have spatial variation; for example, on a rotating sphere it is proportional to the sine of the latitude. Numerical solution of the shallow-water equations presents many (though not all) of the challenges of solving the full three-dimensional atmospheric governing equations, and almost all model development programs include a numerical shallow-water model as a step on the way to a full three-dimensional model.

1.1. Desirable properties. The following properties of the spatial discretization are valuable for ensuring the accuracy of numerical weather and climate models.

1. C-grid staggering. Large-scale extratropical atmospheric flow is close to geostrophic balance, i.e., a balance between the Coriolis and pressure gradient terms, and evolves relatively slowly. The process by which the flow returns towards balance after a disturbance, called geostrophic adjustment, involves the propagation, dispersion, and eventual dissipation of fast inertio-gravity waves. An accurate representation of the geostrophic adjustment process requires that a numerical model capture the propagation of these fast waves sufficiently accurately. In the shallow-water context, and assuming a finite difference or finite volume approach with $\phi$ and $\mathbf{v}$ as the predicted variables, this requires a C-grid staggering and placement of variables, in which $\phi$ is stored at cell centers and a cross-edge component of $\mathbf{v}$ is stored at cell edges. (A similar staggering of variables is useful for simulation of incompressible flow [11].) Other staggerings have less compact approximations to derivatives, and lead to retarded phase speeds and group velocities of the wrong sign for part of the wave spectrum $[1,20]$. The C-grid is usually defined to store normal velocity components at cell edges; below we depart from this for nonorthogonal grids, for the reasons explained in section 4.5 .

2. Mass conservation. Exact or very accurate conservation of mass is considered highly desirable, particularly for long climate simulations, both for the dry component of the air and for moisture and long-lived chemical species transported by the model. The most straightforward way to achieve this is by a flux-form or finite volume discretization of the mass continuity equation.

3. Curl-free pressure gradient. The pressure gradient is one of the largest terms in the governing equations, so small relative errors can have a comparatively large effect. It is particularly important that the pressure gradient should not act as a spurious source of vorticity, since that would contaminate the meteorologically important vortical signal. Thus, a discrete analogue of $\nabla \times \nabla \eta \equiv \mathbf{0}$ for any scalar field $\eta$ is needed.

4. Energy conserving pressure terms. The governing equations of atmospheric flow, in the absence of friction and diabatic heating, conserve the total energy, but they do permit a nonlinear transfer of energy between scales. There is therefore room for debate over whether a numerical model should exactly conserve the resolved energy or permit variations that are meant to model exchanges between resolved and unresolved scales [27]. Nevertheless, a strong argument can be made that the large and essentially linear pressure gradient and Coriolis terms should individually be energy conserving. In the shallow-water context, conservation of energy by the pressure gradient term requires a discrete analogue of $\mathbf{v} \cdot \nabla \phi+\phi \nabla \cdot \mathbf{v} \equiv \nabla \cdot(\mathbf{v} \phi)$. 
5. Energy conserving Coriolis term. The Coriolis force is perpendicular to the velocity. It therefore does no work and does not appear in the energy budget. Conservation of energy by the Coriolis term requires a discrete analogue of $\mathbf{v} \cdot \mathbf{v}^{\perp} \equiv 0$.

6. Steady geostrophic modes. In the case of constant $f$, the continuous shallowwater equations, linearized about a resting basic state with geopotential $\bar{\phi}$, support steady solutions in exact geostrophic balance:

$$
f \mathbf{v}^{\perp}+\nabla \phi=\mathbf{0}
$$

The linearized mass and vorticity equations are

$$
\frac{\partial \phi}{\partial t}+\bar{\phi} \delta=0, \quad \frac{\partial \xi}{\partial t}+f \delta=0
$$

where $\delta=\nabla \cdot \mathbf{v}$ is the velocity divergence; therefore steadiness requires that the flow be divergence-free. In order for a discretization to support steady geostrophic modes, it must imply a discrete analogue of the vorticity equation in which the divergence that appears is zero when $\phi$ is steady. Failure of the discretization to support steady geostrophic modes would imply an inability to represent geostrophic balance - a very undesirable property.

See the review by [26] for a fuller discussion of these issues.

Properties 2, 3, and 4 require the discretization to mimic certain properties of the continuous vector calculus. Schemes with such properties are called mimetic, and there has been much recent progress in developing a deeper theoretical basis for them, as well as frameworks in which they can be systematically constructed (see, e.g., $[18,16,13,14,3,4,8]$ and the references therein). Property 1 , although it is motivated by a desire for accurate wave propagation rather than a desire to mimic the properties of vector calculus, does fit naturally within some of these frameworks. Properties 5 and 6 are of particular relevance to geophysical fluid dynamics and, as far as we are aware, have not been addressed before in the context of mimetic discretizations. Property 5 on its own is nontrivial on a C-grid, even on a latitudelongitude grid [2], because only one component of $\mathbf{v}$ is predicted at each edge so that the relevant component of $\mathbf{v}^{\perp}$ must be reconstructed from components of $\mathbf{v}$ at nearby edges. Property 6 is also challenging on a C-grid because the natural location for $\delta$ is at cell centers, whereas the natural location for $\xi$ is at vertices; thus the divergence that appears in the discrete version of the vorticity equation cannot simply be the natural discrete divergence.

The difficulty of obtaining properties 5 and 6 is illustrated by early attempts to analyze the wave dispersion properties of hexagonal C-grids [17], which found that stationary geostrophic modes were not supported. Subsequently [28], an alternative discretization of the Coriolis term was found that gave properties 5 and 6 on a regular hexagonal grid in planar geometry. This was extended to arbitrary but orthogonal polygonal grids on the sphere by [29,21], and their scheme is the basis of a new weather and climate model being developed at the U.S. National Center for Atmospheric Research and Los Alamos National Laboratory using a hexagonal/pentagonal Voronoi grid (Figure 1.1) [24]. The mixed finite element $P(n) d g-P(n+1)$ family [6] provides an alternative route to developing schemes with these desirable properties.

Mimetic properties are also valuable in the construction of discretizations based on Hamiltonian methods [23, 10, 25], which provide another route to obtaining conservation properties. 

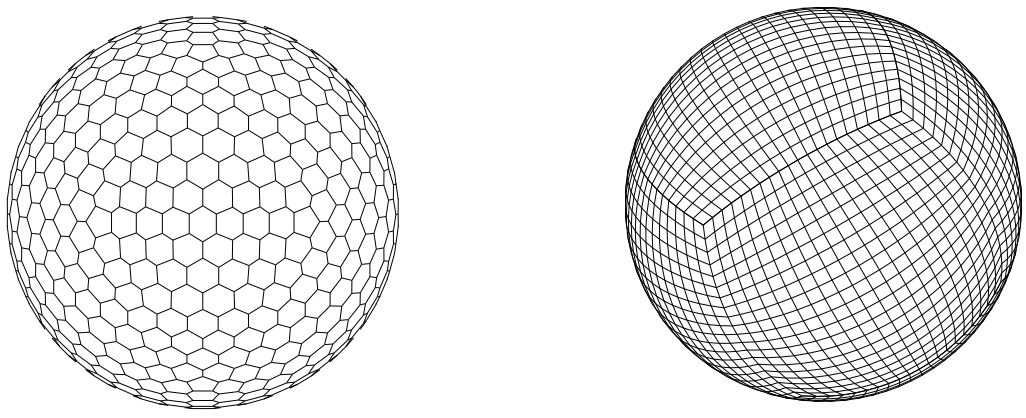

FIG. 1.1. Left: A hexagonal-icosahedral grid generated by a Voronoi tessellation, which has an orthogonal dual. Right: An equiangular cubed sphere grid; all cells are quadrilateral and the grid cell size is quasi-uniform, but it does not have an orthogonal dual.

1.2. The TRiSK scheme and the need for extension. The scheme described by $[29,21]$ has become informally known as TRiSK. TRiSK is applicable to grids whose edges are orthogonal to the edges of a dual grid; this includes VoronoiDelaunay grids, but also orthogonal quadrilateral grids such as the latitude-longitude grid and the conformal cubed sphere [19]. The TRiSK scheme in fact gives all six of the desirable properties listed above. The key to obtaining property 6 is to demand that the vertex divergence that appears in the vorticity equation should be a suitably weighted average of the divergence in the cells adjacent to that vertex; remarkably, there is then a unique way to construct $\mathbf{v}^{\perp}$ to simultaneously satisfy this constraint and energy conservation.

However, the restriction to orthogonal grids is potentially a significant limitation. C-grids that do not have a local logically rectangular quadrilateral structure have a mismatch in the relative numbers of mass, divergence, and vorticity degrees of freedom and so their discrete dispersion relation includes branches of computational modes that have no analogue for the continuous dispersion relation. For example, the hexagonal C-grid supports an extra branch of very-low-frequency Rossby modes [28], while the triangular C-grid supports two extra branches of inertio-gravity modes [7]. A spherical C-grid of kite-shaped quadrilaterals with an orthogonal dual (but not a logically rectangular structure) has recently been proposed, but numerical results indicate that it too supports computational modes [30]. Concern about the possible damaging effects of computational modes (see, e.g., [26]) motivates the search for a suitable quadrilateral grid. However, orthogonal logically rectangular quadrilateral grids on the sphere, such as the latitude-longitude grid and the conformal cubed sphere, have unavoidable singularities, with associated resolution clustering or dilation, so they are considered unsuitable for use on massively parallel computers. Quasi-uniform logically rectangular grids are possible, such as the equiangular cubed sphere [22] (Figure 1.1), but they are not orthogonal. This motivates the development of an extension to TRiSK suitable for nonorthogonal grids.

A further limitation of TRiSK is that, in general, it is no better than first-order accurate, though it approaches second-order on some smooth grids. In the extension to TRiSK described in this paper, all discrete derivative operators are exact when applied to variables integrated over the relevant grid elements (section 2.2), and the only approximations involved are in certain reconstruction and mapping operators, opening up the possibility of extension to higher order. 
1.3. Outline. Section 2 describes the proposed framework for the linearized shallow-water equations in the constant $f$ case on general, not necessarily orthogonal grids, and section 3 demonstrates how the six desirable properties of section 1.1 are obtained. Section 4 describes how the framework can be extended to the nonlinear shallow-water equations with nonconstant $f$. (However, the presentation of specific schemes within the framework is deferred to later work.) These three sections are presented using familiar vector calculus terminology, intended to make the material accessible to the community developing weather and climate models.

Section 5 discusses the prospects for building high-order schemes within the proposed framework. Discrete exterior calculus $[13,14,4,8]$ provides a powerful framework for the construction and theoretical understanding of mimetic discretizations. It is instructive to interpret the present work in the language and framework of discrete exterior calculus, and this is done in section 6 . Section 7 gives the conclusions.

2. Discretization: Linear case. This section describes the discretization of the shallow-water equations, linearized about a resting basic state with constant geopotential $\bar{\phi}$, with constant Coriolis parameter:

$$
\begin{aligned}
\frac{\partial \phi}{\partial t}+\bar{\phi} \nabla \cdot \mathbf{v} & =0, \\
\frac{\partial \mathbf{v}}{\partial t}+f \mathbf{v}^{\perp}+\nabla \phi & =\mathbf{0} .
\end{aligned}
$$

2.1. Notation. Consider a primal grid composed of arbitrary polygons (solid lines in Figure 2.1) and a dual grid (dashed lines) such that each primal cell corresponds to a dual vertex, each primal edge corresponds to a dual edge, and each primal vertex corresponds to a dual cell. There is no requirement for dual edges to be orthogonal to primal edges, nor for dual vertices to have any special placement within the primal cell such as at its centroid. Each cell, edge, and vertex is given a unique index; the corresponding dual vertex, edge, or cell, respectively, has the same index.

Let $\mathbf{n}_{e}$ be a unit normal at edge $e$, and let $\mathbf{t}_{e}=\mathbf{k} \times \mathbf{n}_{e}$ be the unit tangent. Also, let $\mathbf{m}_{e}$ be a unit vector pointing along dual edge $e$ in the same sense as $\mathbf{n}_{e}$, and let $\mathbf{s}_{e}=\mathbf{k} \times \mathbf{m}_{e}$. To keep track of orientations it is useful to define

$$
\begin{aligned}
& n_{e i}=\left\{\begin{aligned}
1 & \text { if } \mathbf{n}_{e}, \mathbf{m}_{e} \text { point out of cell } i \\
-1 & \text { if } \mathbf{n}_{e}, \mathbf{m}_{e} \text { point into cell } i \\
0 & \text { otherwise; }
\end{aligned}\right. \\
& t_{e v}=\left\{\begin{aligned}
1 & \text { if } \mathbf{t}_{e}, \mathbf{s}_{e} \text { point towards vertex } v, \\
-1 & \text { if } \mathbf{t}_{e}, \mathbf{s}_{e} \text { point away from vertex } v, \\
0 & \text { otherwise }
\end{aligned}\right.
\end{aligned}
$$

The discretization works in terms of quantities sampled at the relevant vertices or integrated over the relevant edges or cells. Table 2.1 summarizes the variables used. Lowercase variable names indicate sampled values, while upper case indicates integrated values. Superscript (c) indicates a value at a primal cell or dual vertex, while superscript $(\mathrm{v})$ indicates a value at a primal vertex or dual cell.

Note that we have two different representations of any vector field, one in terms of its "circulation" along dual edges (e.g., $V_{e}$ ), and one in terms of its flux across primal edges (e.g., $\left.U_{e}\right)$. Note also that we have introduced both the point-sampled geopotential $\phi_{i}^{(\mathrm{c})}$ and the cell-integrated geopotential $\Phi_{i}^{(\mathrm{c})}$. This reflects a curious feature of the shallow-water equations, in which $\phi$ plays two roles: in the mass equation 


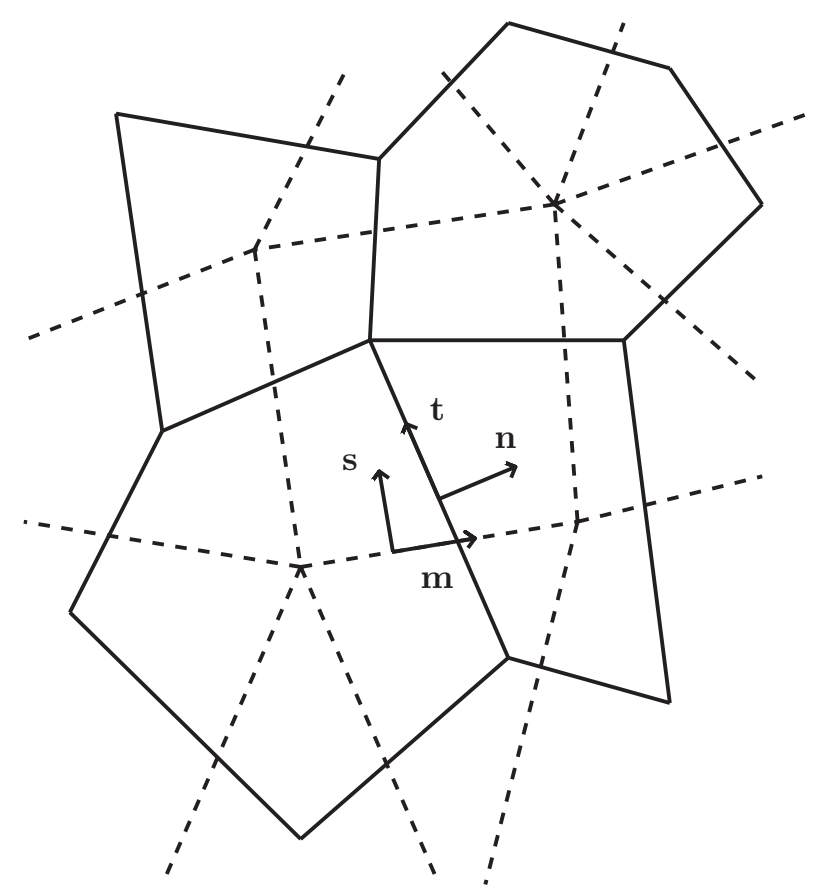

FIG. 2.1. Schematic showing part of an arbitrary polygonal grid and the unit vectors involved in discretizing vector fields. Primal edges are shown as solid lines and dual edges as dashed lines.

it plays the role of a density, for which the natural discretization is the integrated quantity $\Phi_{i}^{(\mathrm{c})}$, while in the momentum equation it plays the role of a pressure, for which the natural discretization is the point-sampled value $\phi_{i}^{(\mathrm{c})}$.

Table 2.2 summarizes the notation used to describe the grid connectivity. This connectivity is used in the definition of the discrete derivative operators in the next subsection.

2.2. Discrete derivative operators. There are four discrete derivative operators available, giving discrete analogues of gradient, divergence, and curl on the primal and dual grids.

1. Gradient integrated along a dual edge.

$$
G_{e} \equiv \int_{\text {dual edge } e} \nabla \phi \cdot \mathbf{m}_{e} d l \equiv-\sum_{i \in \operatorname{CE}(e)} n_{e i} \phi_{i}^{(\mathrm{c})} \equiv\left(\bar{D}_{1} \phi^{(\mathrm{c})}\right)_{e} .
$$

2. Gradient integrated along a primal edge.

$$
\int_{\text {primal edge } e} \nabla \psi \cdot \mathbf{t}_{e} d l \equiv \sum_{v \in \operatorname{VE}(e)} t_{e v} \psi_{v}^{(\mathrm{v})} \equiv\left(D_{1} \psi^{(\mathrm{v})}\right)_{e} .
$$

3. Divergence integrated over a primal cell.

$$
\Delta_{i}^{(\mathrm{c})} \equiv \sum_{e \in \mathrm{EC}(i)} n_{e i} U_{e} \equiv\left(D_{2} U\right)_{i} .
$$


TABLE 2.1

Summary of the variables used in the discretization. Lowercase variable names indicate sampled values, while upper case indicates integrated values. Superscript (c) indicates a value at a primal cell or dual vertex, while superscript (v) indicates a value at a primal vertex or dual cell.

\begin{tabular}{|c|c|c|}
\hline \begin{tabular}{|l} 
Discrete \\
variable \\
\end{tabular} & Integral definition & Description \\
\hline $\begin{array}{l}\phi_{i}^{(\mathrm{c})} \\
\chi_{i}^{(\mathrm{c})}, \chi_{v}^{(\mathrm{v})} \\
\psi_{i}^{(\mathrm{c})}, \psi_{v}^{(\mathrm{v})}\end{array}$ & & $\begin{array}{l}\text { Geopotential sampled at dual ver- } \\
\text { tex } i \\
\text { Velocity potential sampled at dual } \\
\text { vertex } i \text { or primal vertex } v \\
\text { Stream function sampled at dual } \\
\text { vertex } i \text { or primal vertex } v\end{array}$ \\
\hline $\begin{array}{l}V_{e} \\
U_{e} \\
V_{e}^{\perp} \\
U_{e}^{\perp} \\
C_{e} \\
F_{e} \\
C_{e}^{\perp} \\
F_{e}^{\perp} \\
G_{e}\end{array}$ & 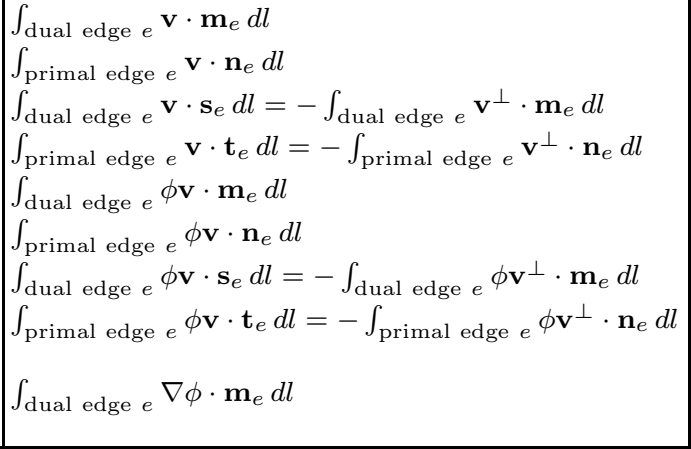 & $\begin{array}{l}\text { Circulation along dual edge } e \\
\text { Volume flux across primal edge } e \\
\text { Volume flux across dual edge } e \\
\text { Circulation along primal edge } e \\
\text { Mass circulation along dual edge } e \\
\text { Mass flux across primal edge } e \\
\text { Mass flux across a dual edge } e \\
\text { Mass circulation along primal edge } \\
e \\
\text { Geopotential gradient integrated } \\
\text { along dual edge } e\end{array}$ \\
\hline$\Phi_{i}^{(c)}$ & $\int_{\text {primal cell } i} \phi d A$ & $\begin{array}{l}\text { Geopotential integrated over pri- } \\
\text { mal cell } i\end{array}$ \\
\hline$\Phi_{v}^{(v)}$ & $\int_{\text {dual cell } v} \phi d A$ & $\begin{array}{l}\text { Geopotential integrated over dual } \\
\text { cell } v\end{array}$ \\
\hline$\Delta_{i}^{(\mathrm{c})}$ & $\int_{\text {primal cell } i} \nabla \cdot \mathbf{v} d A$ & $\begin{array}{l}\text { Divergence integrated over primal } \\
\text { cell } i\end{array}$ \\
\hline$\Delta_{v}^{(\mathrm{v})}$ & $\int_{\text {dual cell } v} \nabla \cdot \mathbf{v} d A=\int_{\text {dual cell } v} \mathbf{k} \cdot \nabla \times \mathbf{v}^{\perp} d A$ & $\begin{array}{l}\text { Divergence integrated over dual } \\
\text { cell } v\end{array}$ \\
\hline$\Xi_{v}^{(\mathrm{v})}$ & $\int_{\text {dual cell } v} \mathbf{k} \cdot \nabla \times \mathbf{v} d A$ & $\begin{array}{l}\text { Relative vorticity integrated over } \\
\text { dual cell } v\end{array}$ \\
\hline$\Xi_{i}^{(\mathrm{c})}$ & $\int_{\text {primal cell } i} \mathbf{k} \cdot \nabla \times \mathbf{v} d A=-\int_{\text {primal cell } i} \nabla \cdot \mathbf{v}^{\perp} d A$ & $\begin{array}{l}\text { Relative vorticity integrated over } \\
\text { primal cell } i\end{array}$ \\
\hline$Z_{v}^{(\mathrm{v})}$ & $\int_{\text {dual cell } v} \zeta d A$ & $\begin{array}{l}\text { Absolute vorticity integrated over } \\
\text { dual cell } v\end{array}$ \\
\hline
\end{tabular}

TABLE 2.2

Summary of notation used to describe the grid connectivity.

\begin{tabular}{|l|l|}
\hline Edges of primal cell $i$ & $\mathrm{EC}(i)$ \\
Edges incident on primal vertex $v$ & $\mathrm{EV}(v)$ \\
Primal cells either side of primal edge $e$ & $\mathrm{CE}(e)$ \\
Primal vertices at the ends of primal edge $e$ & $\mathrm{VE}(e)$ \\
Primal cells surrounding primal vertex $v$ & $\mathrm{CV}(v)$ \\
Vertices of primal cell $i$ & $\mathrm{VC}(i)$ \\
\hline
\end{tabular}


4. Curl integrated over a dual cell.

$$
\Xi_{v}^{(\mathrm{v})} \equiv \sum_{e \in \mathrm{EV}(v)} t_{e v} V_{e} \equiv\left(\bar{D}_{2} V\right)_{e}
$$

Here, $\bar{D}_{1}, D_{1}, D_{2}, \bar{D}_{2}$ are the matrix representations of the derivative operators defined by these equations. See section 3.5 for further discussion. Note also that $\Xi_{i}^{(\mathrm{c})}=$ $\left(D_{2} U^{\perp}\right)_{i}$ and $\Delta_{v}^{(\mathrm{v})}=\left(-\bar{D}_{2} V^{\perp}\right)_{v}$. All of these operators have simple expressions in terms of appropriately signed sums. Moreover, because the discrete variables are defined to be integrals over the relevant grid elements, these derivative operators are all exact: (2.5) and (2.6) are exact instances of the one-dimensional fundamental theorem of calculus on a manifold, while (2.7) and (2.8) are exact instances of the divergence theorem and Stokes's theorem.

2.3. Discrete mass and momentum equations. Integrating (2.1) over a primal cell and (2.2) dotted with $\mathbf{m}_{e}$ along a dual edge leads to the discrete mass and momentum equations:

$$
\begin{aligned}
\dot{\Phi}_{i}^{(\mathrm{c})}+\bar{\phi} \sum_{e \in \mathrm{EC}(i)} n_{e i} U_{e} & =0, \\
\dot{V}_{e}-f V_{e}^{\perp}-\sum_{i \in \operatorname{CE}(e)} n_{e i} \phi_{i}^{(\mathrm{c})} & =0 .
\end{aligned}
$$

Here the dot above a symbol means a time derivative.

In order to integrate these equations in time we need to be able to reconstruct $\phi_{i}^{(\mathrm{c})}, U_{e}$, and $V_{e}^{\perp}$ from the predicted variables $\Phi_{i}^{(c)}$ and $V_{e}$. This is discussed in the next subsection.

2.4. Reconstruction and mapping operators. By taking suitable linear combinations of the predicted variables we can reconstruct the other variables that are needed by the discretization. Let

$$
\begin{gathered}
\phi_{i}^{(\mathrm{c})}=\sum_{i^{\prime} \in \mathrm{I}(i)} I_{i i^{\prime}} \Phi_{i^{\prime}}^{(\mathrm{c})}, \\
\xi_{v}^{(\mathrm{v})}=\sum_{v^{\prime} \in \mathrm{J}(v)} J_{v v^{\prime}} \Xi_{v^{\prime}}^{(\mathrm{v})}, \\
U_{e}=\sum_{e^{\prime} \in \mathrm{H}(e)} H_{e e^{\prime}} V_{e^{\prime}},
\end{gathered}
$$

and

$$
V_{e}^{\perp}=\sum_{e^{\prime} \in \mathrm{W}(e)} W_{e e^{\prime}} U_{e^{\prime}}
$$

Here, $I_{i i^{\prime}}, J_{v v^{\prime}}, H_{e e^{\prime}}$, and $W_{e e^{\prime}}$ are weights that define the operators, and $\mathrm{I}(i), \mathrm{J}(v)$, $\mathrm{H}(e)$, and $\mathrm{W}(e)$ are the corresponding stencils. (For the theoretical development below it is convenient to regard the stencils as global with most of the weights equal to zero, allowing us to omit explicit specification of the stencil and express the operators as 
matrix multiplication. However, any practical implementation would make use of local stencils.) For now we leave the weights unspecified; demanding mimetic properties will impose certain constraints on the weights.

In order to determine suitable values of $W_{e e^{\prime}}$ it is necessary to introduce a mapping from primal cells to dual cells:

$$
\Phi_{v}^{(\mathrm{v})}=\sum_{i \in \mathrm{CV}(v)} R_{v i} \Phi_{i}^{(\mathrm{c})} .
$$

The weights $R_{v i}$ should satisfy

$$
\sum_{v \in \mathrm{VC}(i)} R_{v i}=1
$$

in order to preserve the global integral of $\phi$, and

$$
\frac{1}{A_{v}^{(\mathrm{v})}} \sum_{i \in \mathrm{CV}(v)} R_{v i} A_{i}^{(\mathrm{c})}=1,
$$

where $A_{i}^{(\mathrm{c})}$ is the area of primal cell $i$ and $A_{v}^{(\mathrm{v})}$ is the area of dual cell $v$, in order to preserve a constant value of $\phi$. The relation between $R$ and $W$ will be explained in section 3.4.

Note that, unlike the discrete derivative operators, these reconstruction and mapping operators can only be approximate.

3. Properties of the discretization. The discretization described above is manifestly C-grid based (property 1 ) and is clearly mass conserving ((2.9), property 2 ). In the following subsections we show that it also satisfies properties 3 to 6 , provided the reconstruction and mapping operators satisfy certain constraints, and some further properties of the discretization are established.

3.1. Vanishing curl of gradient. Consider $\phi_{i}^{(\mathrm{c})}$, and compute the curl of its gradient using (2.5) and (2.8):

$$
-\sum_{e \in \operatorname{EV}(v)} \sum_{i \in \operatorname{CE}(e)} t_{e v} n_{e i} \phi_{i}^{(\mathrm{c})} .
$$

It is easily verified that each $\phi_{i}^{(\mathrm{c})}$ appears twice, once with each sign; therefore all contributions cancel and the curl of the gradient is identically zero.

Similarly, consider $\psi_{v}^{(\mathrm{v})}$, and compute the curl of its gradient using (2.6) and (2.7):

$$
\sum_{e \in \mathrm{EC}(i)} \sum_{v \in \mathrm{VE}(e)} n_{e i} t_{e v} \psi_{v}^{(\mathrm{v})} .
$$

Again all contributions cancel and the curl of the gradient is identically zero.

3.2. Energy conserving $\phi$ terms. For the continuous linearized shallow-water equations the total energy is given by

$$
E=\frac{1}{2}(\phi, \phi)+\frac{\bar{\phi}}{2}(\mathbf{v}, \mathbf{v}),
$$


where the inner products are defined as

$$
\left(\phi_{(1)}, \phi_{(2)}\right)=\int \phi_{(1)} \phi_{(2)} d A, \quad\left(\mathbf{v}_{(1)}, \mathbf{v}_{(2)}\right)=\int \mathbf{v}_{(1)} \cdot \mathbf{v}_{(2)} d A
$$

for scalar fields $\phi_{(1)}$ and $\phi_{(2)}$ and vector fields $\mathbf{v}_{(1)}$ and $\mathbf{v}_{(2)}$, respectively. Taking the inner product of $\phi$ with the mass equation and $\phi \mathbf{v}$ with the momentum equation and using the symmetry of the inner product gives the total energy tendency:

$$
\dot{E}=-\bar{\phi}(\phi, \nabla \cdot \mathbf{v})-f \bar{\phi}\left(\mathbf{v}, \mathbf{v}^{\perp}\right)-\bar{\phi}(\mathbf{v}, \nabla \phi) .
$$

The sum of the first and third terms on the right-hand side reduces to a boundary integral, which vanishes under suitable boundary conditions or for a boundaryless domain such as the surface of the sphere. In other words, the gradient is minus the adjoint of the divergence with respect to these inner products.

In order for the discretization to have energy conserving $\phi$ terms, we require a discrete analogue of this adjoint property. The appropriate discrete inner products are

$$
\begin{aligned}
\left(\Phi_{(1)}^{(c)}, \Phi_{(2)}^{(c)}\right)_{I} & =\sum_{i, i^{\prime}} \Phi_{(1) i^{\prime}}^{(c)} I_{i^{\prime} i} \Phi_{(2) i}^{(c)}, \\
\left(\phi_{(1)}^{(c)}, \phi_{(2)}^{(c)}\right)_{I^{-1}} & =\sum_{i, i^{\prime}} \phi_{(1) i}^{(c)}\left(I^{-1}\right)_{i i^{\prime}} \phi_{(2) i^{\prime}}^{(c)}
\end{aligned}
$$

and

$$
\begin{aligned}
\left(V_{(1)}, V_{(2)}\right)_{H} & =\sum_{e, e^{\prime}} V_{(1) e} H_{e e^{\prime}} V_{(2) e^{\prime}}, \\
\left(U_{(1)}, U_{(2)}\right)_{H^{-1}} & =\sum_{e, e^{\prime}} U_{(1) e}\left(H^{-1}\right)_{e e^{\prime}} U_{(2) e^{\prime}},
\end{aligned}
$$

implying the identities

$$
\begin{aligned}
& \left(\Phi_{(1)}^{(c)}, \Phi_{(2)}^{(c)}\right)_{I}=\left(\phi_{(1)}^{(c)}, \phi_{(2)}^{(c)}\right)_{I^{-1}}=\sum_{i} \Phi_{(1) i}^{(c)} \phi_{(2) i}^{(c)}, \\
& \left(V_{(1)}, V_{(2)}\right)_{H}=\left(U_{(1)}, U_{(2)}\right)_{H^{-1}}=\sum_{e} V_{(1) e} U_{(2) e} .
\end{aligned}
$$

In order for the inner products to be symmetric and positive definite, the matrices $I$ and $H$ must be symmetric and positive definite. Then their respective inverses exist and are also symmetric and positive definite. However, the inverses will generally be full (not sparse) matrices unless $I$ and $H$ are diagonal. In an analogous way, we can define an inner product for primal vertex or dual cell scalars, provided $J$ is symmetric and positive definite.

The discrete approximation to the energy is

$$
E=\frac{1}{2}\left(\Phi^{(\mathrm{c})}, \Phi^{(\mathrm{c})}\right)_{I}+\frac{\bar{\phi}}{2}(V, V)_{H},
$$

and, using the symmetry of $I$ and $H$,

$$
\dot{E}=-\bar{\phi}\left(\Phi^{(\mathrm{c})}, \Delta^{(\mathrm{c})}\right)_{I}+f \bar{\phi}\left(V, V^{\perp}\right)_{H}-\bar{\phi}(V, G)_{H} .
$$


The first inner product on the right-hand side is

$$
\begin{aligned}
\left(\Phi^{(\mathrm{c})}, \Delta^{(\mathrm{c})}\right)_{I} & =\sum_{i} \phi_{i}^{(c)} \Delta_{i}^{(\mathrm{c})}=\sum_{i} \phi_{i}^{(c)} \sum_{e} n_{e i} U_{e} \\
& =\sum_{e} U_{e} \sum_{i} n_{e i} \phi_{i}=-(V, G)_{H}
\end{aligned}
$$

(ignoring boundary contributions). Thus the discrete gradient is minus the adjoint of the discrete divergence with respect to the discrete inner products defined above. The $\phi$ terms will therefore conserve energy.

3.3. Energy conserving Coriolis term. The middle term on the right-hand side of (3.5) vanishes identically, so the Coriolis term is energy conserving for the continuous equations. In order for the discrete Coriolis term to be energy conserving we require a discrete analogue of this vanishing inner product:

$$
\left(V, V^{\perp}\right)_{H}=\sum_{e} U_{e} V_{e}^{\perp}=\sum_{e} \sum_{e^{\prime}} U_{e} W_{e e^{\prime}} U_{e^{\prime}}=0 .
$$

In order for this to hold for arbitrary velocity fields, $W_{e e^{\prime}}$ must be antisymmetric.

3.4. Stationary geostrophic modes. The scheme will support stationary geostrophic modes, provided that steady vanishing divergence implies that all variables are steady. Here we establish the constraints needed to ensure that vanishing divergence $\left(\Delta_{i}^{(\mathrm{c})}=0\right)$ implies steady vorticity. Applying (2.8) to (2.10) gives the discrete vorticity equation:

$$
\dot{\Xi}_{v}^{(\mathrm{v})}+f \Delta_{v}^{(\mathrm{v})}=0 .
$$

Thus we require that $\Delta_{i}^{(\mathrm{c})}=0 \forall i \Rightarrow \Delta_{v}^{(\mathrm{v})}=0 \forall v$. This can be achieved by demanding that $\Delta_{v}^{(\mathrm{v})}$ is given by the mapping (2.15):

$$
\Delta_{v}^{(\mathrm{v})}=\sum_{i \in \mathrm{CV}(v)} R_{v i} \Delta_{i}^{(\mathrm{c})} \forall v .
$$

Expressing both sides of this equation in terms of $U_{e}$ using (2.8) and (2.14) on the left-hand side and (2.7) on the right-hand side gives

$$
-\sum_{e} \sum_{e^{\prime}} t_{e v} W_{e e^{\prime}} U_{e^{\prime}}=\sum_{i} \sum_{e^{\prime}} R_{v i} n_{e^{\prime} i} U_{e^{\prime}} \quad \forall v .
$$

This must hold for arbitrary $U_{e^{\prime}}$, so

$$
-\sum_{e} t_{e v} W_{e e^{\prime}}=\sum_{i} R_{v i} n_{e^{\prime} i} \quad \forall v, e^{\prime} .
$$

Thus we require values of $W_{e e^{\prime}}$ that are antisymmetric and satisfy the constraint (3.17) relating $W$ to $R$.

In fact, these are algebraically identical to the constraints that are satisfied by the analogous weights in the TRiSK scheme (apart from a different sign convention for the Coriolis term in [29]). Therefore, provided $R$ satisfies (2.16), there is a unique solution for the weights $W$ in terms of $R$, given explicitly (up to a sign change) by $[29$, section 2]. 
Combining the vorticity equation (3.14) with the dual-cell mass equation

$$
\dot{\Phi}_{v}^{(\mathrm{v})}+\bar{\phi} \Delta_{v}^{(\mathrm{v})}=0
$$

shows that a discrete analogue of the linearized potential vorticity $\Xi_{v}^{(\mathrm{v})} / \bar{\phi}-f \Phi_{v}^{(\mathrm{v})} / \bar{\phi}^{2}$ is steady. This, in turn, implies that the linearized potential enstrophy is globally conserved. Conservation of potential enstrophy is often argued to be a desirable property of a discretization (see, e.g., [23, 10], but also [27]). However, it appears unlikely that both energy and potential enstrophy conservation can be obtained in the general nonlinear case within the present framework. Therefore, we do not consider potential enstrophy conservation further in this paper.

3.5. Compact notation. From now on it will be convenient to use a compact notation in which the four discrete derivatives (2.5)-(2.8) are represented by the matrices $\bar{D}_{1}, D_{1}, D_{2}, \bar{D}_{2}$, respectively, and the action of the various derivative and mapping operators will be expressed as matrix multiplication without explicitly writing sums over indices. For the derivative operators, subscript 1 indicates that the result of the operator is a quantity integrated over a one-dimensional grid element (a primal or dual edge), while subscript 2 indicates that the result is a quantity integrated over a two-dimensional grid element (a primal or dual cell). An overbar means that the operator acts on dual grid elements; no overbar means it acts on primal grid elements. In this compact notation, $D_{2}=-\bar{D}_{1}^{\mathrm{T}}, \bar{D}_{2}=D_{1}{ }^{\mathrm{T}}$, the results of section 3.1 can be summarized as $\bar{D}_{2} \bar{D}_{1} \equiv 0, D_{2} D_{1} \equiv 0$, while section 3.4 requires $W$ to satisfy $-\bar{D}_{2} W=R D_{2}$, implying, among other things, that $\bar{D}_{2} W D_{1}=-R D_{2} D_{1} \equiv 0$.

3.6. Discrete scalar and vector Laplacians. There are four discrete versions of the scalar Laplacian, depending on whether the quantity to be differentiated resides at primal vertices or dual vertices (for point values) or primal cells or dual cells (for integrated quantities).

Consider first a field of point values defined at dual vertices $\phi^{(\mathrm{c})}$. To compute its Laplacian we must compute its gradient (2.5), convert the gradient from a dual edge integral to a primal edge integral (2.13), compute the divergence of the gradient (2.7), and convert the result from a cell integral to a point value (2.11). The discrete Laplacian is therefore

$$
\nabla^{2} \phi^{(\mathrm{c})} \approx I D_{2} H \bar{D}_{1} \phi^{(\mathrm{c})} .
$$

Proceeding in a similar way for the other types of scalar data gives

$$
\begin{gathered}
\nabla^{2} \psi^{(\mathrm{v})} \approx-J \bar{D}_{2} H^{-1} D_{1} \psi^{(\mathrm{v})}, \\
\nabla^{2} \Phi^{(\mathrm{c})} \approx D_{2} H \bar{D}_{1} I \Phi^{(\mathrm{c})}, \\
\nabla^{2} \Xi^{(\mathrm{v})} \approx-\bar{D}_{2} H^{-1} D_{1} J \Xi^{(\mathrm{v})},
\end{gathered}
$$

where, in (3.21) and (3.22), $\nabla^{2}$ is shorthand for the cell integral of the Laplacian of the variable whose cell integral is its argument. It is easily verified that these Laplacian operators are self-adjoint and negative semidefinite under the appropriate inner product.

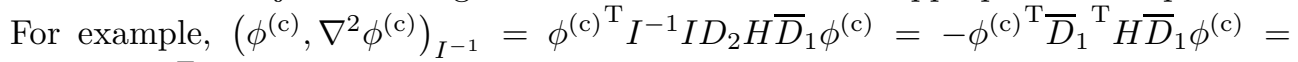
$-\left(\bar{D}_{1} \phi^{(\mathrm{c})}\right)^{\mathrm{T}} H\left(\bar{D}_{1} \phi^{(\mathrm{c})}\right)$, which vanishes for $\phi^{(\mathrm{c})}$ in the null space of $\bar{D}_{1}$, i.e., for constant $\phi^{(\mathrm{c})}$, and is negative otherwise. 
The scalar Laplacian arises when a wave equation is derived from the linearized shallow-water equations. Relatedly, it also arises as part of a Helmholtz operator when a semi-implicit time integration scheme is used. Finally, a discrete Laplacian (or iterated Laplacian) may be useful for applying scale-selective dissipation to a scalar field.

A natural discretization of the vector Laplacian is most easily derived by writing

$$
\begin{aligned}
\nabla^{2} \mathbf{v} & =\nabla(\nabla \cdot \mathbf{v})-\nabla \times(\nabla \times \mathbf{v}) \\
& =\nabla(\nabla \cdot \mathbf{v})+\mathbf{k} \times \nabla(\mathbf{k} \cdot \nabla \times \mathbf{v}) .
\end{aligned}
$$

First consider the discrete Laplacian of a dual-edge-integrated vector $V$. To compute the first term on the right-hand side of (3.23), we must convert to a primal-edgeintegrated vector (2.13), compute the divergence (2.7), convert the cell integrated value to a point value (2.11), and compute the gradient (2.5). To compute the second term on the right-hand side of (3.23), we must compute the curl (2.8), convert the dual-cell-integrated value to a point value (2.12), compute the gradient (2.6), then convert back to a dual-edge-integrated value (the inverse of (2.13)), making appropriate allowance for signs. Thus, the discrete vector Laplacian is

$$
\nabla^{2} V \approx \bar{D}_{1} I D_{2} H V-H^{-1} D_{1} J \bar{D}_{2} V .
$$

Similarly, for a primal-edge-integrated vector the discrete Laplacian is given by

$$
\nabla^{2} U \approx H \bar{D}_{1} I D_{2} U-D_{1} J \bar{D}_{2} H^{-1} U .
$$

Again these operators are self-adjoint and either negative semidefinite (if the domain topology supports a null space) or negative definite.

A discrete vector Laplacian (or iterated Laplacian) may be useful for applying a scale-selective dissipation to the velocity field. A scale-selective dissipation of this form has been used on a hexagonal/pentagonal Voronoi spherical grid with an orthogonal dual, for which diagonal versions of $I, J$, and $H$ are appropriate (Todd Ringler and Joe Klemp, personal communication).

3.7. Discrete Helmholtz-Hodge decomposition. Nicolaides [18] showed that, for orthogonal C-grids, and given suitable boundary conditions, there is a unique decomposition of a discrete vector field into divergent and rotational components, and these two components are orthogonal in the appropriate discrete inner product. (The assumptions made by [18] rule out the possibility of a third, harmonic, component, which would be both irrotational and nondivergent.)

The analogous result for nonorthogonal grids in the present framework is

$$
\begin{aligned}
V & =\bar{D}_{1} \chi^{(\mathrm{c})}-H^{-1} D_{1} \psi^{(\mathrm{v})} \\
\text { or } \quad U & =H \bar{D}_{1} \chi^{(\mathrm{c})}-D_{1} \psi^{(\mathrm{v})},
\end{aligned}
$$

where the vorticity and divergence are related to the stream function and velocity potential, respectively, by the scalar Laplacian operators of section 3.6:

$$
\begin{aligned}
& \Xi^{(\mathrm{v})}=\bar{D}_{2} V=-\bar{D}_{2} H^{-1} D_{1} \psi^{(\mathrm{v})}, \\
& \Delta^{(\mathrm{c})}=D_{2} U=D_{2} H \bar{D}_{1} \chi^{(\mathrm{c})} .
\end{aligned}
$$

(Here too we have ignored the possibility of a harmonic component of the vector field; this is appropriate on the surface of a sphere, but would not be so, for example, on 
a doubly periodic plane, which is topologically a torus, where a constant velocity field would have zero divergence and zero curl.) The proof of this decomposition is a straightforward extension of that given by [18], using the fact that $I, J$, and $H$ are invertible, and is not repeated here. (A more compact proof is possible using discrete exterior calculus; see section 6 and [8].)

A similar decomposition is possible for $V^{\perp}$ and $U^{\perp}$ :

$$
\begin{aligned}
V^{\perp} & =H^{-1} D_{1} \chi^{(\mathrm{v})}+\bar{D}_{1} \psi^{(\mathrm{c})}, \\
U^{\perp} & =D_{1} \chi^{(\mathrm{v})}+H \bar{D}_{1} \psi^{(\mathrm{c})},
\end{aligned}
$$

where

$$
\begin{aligned}
& \Xi^{(\mathrm{c})}=D_{2} U^{\perp}=D_{2} H \bar{D}_{1} \psi^{(\mathrm{c})}, \\
& \Delta^{(\mathrm{v})}=-\bar{D}_{2} V^{\perp}=-\bar{D}_{2} H^{-1} D_{1} \chi^{(\mathrm{v})} .
\end{aligned}
$$

3.8. Operator map. Figure 3.1 summarizes the operators used in the discretization and how they relate the variables to each other. It is illuminating to trace around the operator map the paths defined by the sequences of operators involved in the scalar and vector Laplacians (section 3.6) and the Helmholtz-Hodge decompositions (section 3.7).

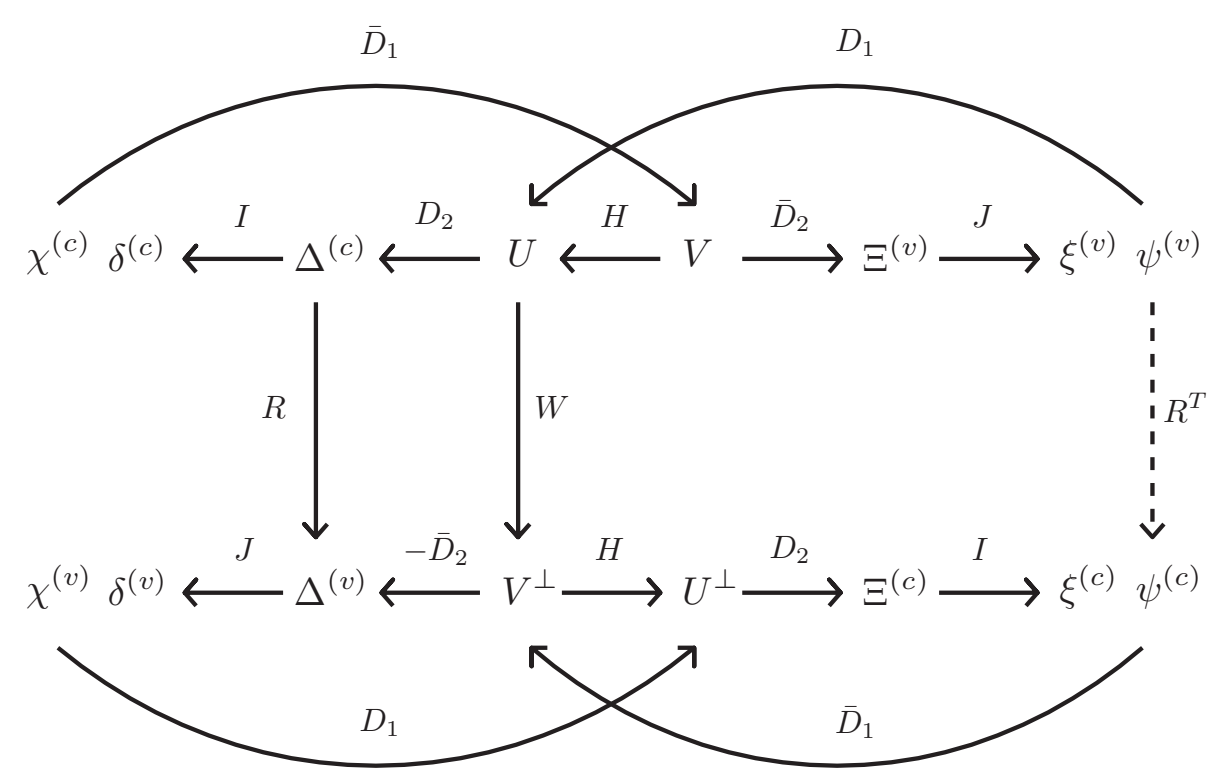

FIG. 3.1. Summary of the operators used in the discretization.

Note that the operators $R$ and $W$ involve some form of averaging to transfer variables from the primal grid to the dual grid. Thus, the variables in the bottom row are averaged versions of those in the top row. The operators $R$ and $W$ are not necessarily invertible. Thus, the variables in the bottom row can be constructed from those in the top row, but not necessarily the converse. The loss of accuracy due to the need for averaging in the Coriolis term is an unavoidable limitation of C-grid staggering.

The mapping $W$ is constructed so that $-\bar{D}_{2} W=R D_{2}$, so that the dual cell divergence is a suitably averaged version of the primal cell divergence. This is the 
key to obtaining property 6 . One might wonder if the mapping $W$ also implies some relation between the dual cell vorticity $\Xi^{(\mathrm{v})}$ and the primal cell vorticity $\Xi^{(\mathrm{c})}$. In fact, provided $\Delta^{(\mathrm{c})}=0$, it implies that the dual vertex stream function $\psi^{(\mathrm{c})}$ is an interpolated version of the primal vertex stream function $\psi^{(\mathrm{v})}$, with the interpolation given by the transpose $R^{\mathrm{T}}$, as we show next. However, if $\Delta^{(\mathrm{c})} \neq 0$, then $\psi^{(\mathrm{c})}$ will also have a contribution from $\chi^{(\mathrm{c})}$.

If $\Delta^{(\mathrm{c})}=0$, then $\bar{D}_{1} \chi^{(\mathrm{c})}=0$, and $V^{\perp}$ may be expressed as $V^{\perp}=W U=$ $-W D_{1} \psi^{(\mathrm{v})}$. Then, using the fact that $W D_{1}=\left(-\bar{D}_{2} W\right)^{\mathrm{T}}=\left(R D_{2}\right)^{\mathrm{T}}=-\bar{D}_{1} R^{\mathrm{T}}$, we have

$$
V^{\perp}=\bar{D}_{1} R^{\mathrm{T}} \psi^{(\mathrm{v})}
$$

At the same time, $\Delta^{(\mathrm{c})}=0$ also implies $\Delta^{(\mathrm{v})}=0$ and $D_{1} \chi^{(\mathrm{v})}=0$; then (3.30) reduces to

$$
V^{\perp}=\bar{D}_{1} \psi^{(\mathrm{c})}
$$

Comparison with (3.34) then gives

$$
\psi^{(\mathrm{c})}=R^{\mathrm{T}} \psi^{(\mathrm{v})},
$$

up to an additive constant (the constant being of no physical significance).

It is interesting to note, from the symmetry of the operator map (Figure 3.1), that, instead of the maps $R$ and $W$, we could have chosen instead to construct, say, maps $\widetilde{R}$ and $\widetilde{W}$, such that

$$
U^{\perp}=\widetilde{W} V, \quad \Xi^{(\mathrm{c})}=\widetilde{R} \Xi^{(\mathrm{v})}, \quad D_{2} \widetilde{W}=\widetilde{R} \bar{D}_{2} .
$$

However, this property is less useful for the shallow-water problem than the property 6 that we obtain using the $R$ and $W$ maps.

4. Nonlinear case. The extension of the above scheme to the nonlinear shallowwater equations with variable $f$ closely follows that in [21] for the TRiSK scheme. The nonlinear continuous equations are rewritten as

$$
\begin{aligned}
\frac{\partial \phi}{\partial t}+\nabla \cdot \mathbf{F} & =0 \\
\frac{\partial \mathbf{v}}{\partial t}+\mathbf{Q}^{\perp}+\nabla(\phi+k) & =\mathbf{0},
\end{aligned}
$$

where $\mathbf{F}=\mathbf{v} \phi$ is the mass flux, $\mathbf{Q}=q \mathbf{F}$ is the potential vorticity flux, where $q=\zeta / \phi$ is the potential vorticity, and $k=|\mathbf{v}|^{2} / 2$ is the kinetic energy per unit mass.

The curl of the momentum equation gives the vorticity equation, which we write here as a conservation law for the potential vorticity:

$$
\frac{\partial}{\partial t}(\phi q)+\nabla \cdot \mathbf{Q}=0 .
$$

The potential vorticity is dynamically important because it is materially conserved, and it determines the balanced component of the flow through the invertibility principle (see, e.g., [15]). It is therefore highly desirable to have a discrete analogue of (4.3) that is compatible with the discrete mass equation (see section 4.2). 
The nonlinear energy equation is obtained by taking the inner product of $\phi+k$ with (4.1) plus the inner product of $\mathbf{F}$ with (4.2):

$$
\dot{E}=-(\phi+k, \nabla \cdot \mathbf{F})-\left(\mathbf{F}, q \mathbf{F}^{\perp}\right)-(\mathbf{F}, \nabla(\phi+k))=0,
$$

where

$$
E=\frac{1}{2}(\phi, \phi)+\frac{1}{2}(\mathbf{F}, \mathbf{v})=\int \frac{1}{2} \phi^{2}+\frac{1}{2} \phi|\mathbf{v}|^{2} d A .
$$

4.1. Discrete nonlinear equations. Introduce the discrete mass circulation $C$ and mass flux $F$ (Table 2.1), with $F=H C, C^{\perp}=W F$, and $F^{\perp}=H C^{\perp}$. The discrete nonlinear equations are then

$$
\begin{aligned}
\dot{\Phi}_{i}^{(\mathrm{c})}+\left(D_{2} F\right)_{i} & =0, \\
\dot{V}_{e}-Q_{e}^{\perp}+\left(\bar{D}_{1}(\phi+k)\right)_{e} & =0 .
\end{aligned}
$$

In addition to the operators needed for the discrete linear equations, we now need operators to construct the discrete mass flux across primal edges $F_{e}$, the discrete potential vorticity flux across dual edges $Q_{e}^{\perp}$, and the kinetic energy per unit mass at dual vertices $k_{i}^{(\mathrm{c})}$.

Let

$$
F_{e}=\sum_{e^{\prime}} H_{e e^{\prime}} \hat{\phi}_{e e^{\prime}} V_{e^{\prime}}
$$

for some weights $\hat{\phi}_{e e^{\prime}}$, symmetric in $\left\{e, e^{\prime}\right\}$, to be constructed from dual vertex values $\phi_{i}^{(\mathrm{c})}$. Also, let $k^{(\mathrm{c})}=I K^{(\mathrm{c})}$, where

$$
K_{i}^{(\mathrm{c})}=\sum_{e} \sum_{e^{\prime}} T_{i e e^{\prime}} V_{e} V_{e^{\prime}}
$$

for some weights $T_{i e e^{\prime}}$. The requirement for energy conservation will determine a relation between $\hat{\phi}_{e e^{\prime}}$ and $T_{i e e^{\prime}}$.

The construction of $Q_{e}^{\perp}$ will be discussed in section 4.4.

4.2. Discrete potential vorticity equation. Applying (2.15) to (4.6) and applying (2.8) to (4.7) gives the dual cell mass and vorticity equations

$$
\begin{aligned}
& \dot{\Phi}_{v}^{(\mathrm{v})}-\left(\bar{D}_{2} C^{\perp}\right)_{v}^{(\mathrm{v})}=0, \\
& \dot{Z}_{v}^{(\mathrm{v})}-\left(\bar{D}_{2} Q^{\perp}\right)_{v}^{(\mathrm{v})}=0 .
\end{aligned}
$$

Define the primal vertex potential vorticity $q_{v}^{(\mathrm{v})}$ by

$$
Z_{v}^{(\mathrm{v})}=q_{v}^{(\mathrm{v})} \Phi_{v}^{(\mathrm{v})}
$$

Then (4.11) is a discrete analogue of the potential vorticity equation (4.3). It is compatible with the dual cell mass equation in the following sense: if the potential vorticity field is constant, $q_{v}^{(\mathrm{v})}=q=$ const, say, and $Q_{e}^{\perp}=q C_{e}^{\perp}$, then $\dot{q}_{v}^{(\mathrm{v})}=0$. 
4.3. Energy conserving $\phi$ terms. By analogy with the continuous case, take the inner product of $(4.6)$ with $\Phi^{(\mathrm{c})}+K^{(\mathrm{c})}$ plus the inner product of (4.7) with $C$. The terms not involving time derivatives reduce to

$$
-\left(\Phi^{(\mathrm{c})}+K^{(\mathrm{c})}, D_{2} F\right)_{I}-\left(C, Q^{\perp}\right)_{H}-\left(C, \bar{D}_{1}(\phi+k)\right)_{H} .
$$

By the properties of the discrete derivatives, the first and third terms sum to zero (ignoring any boundary contributions). The middle term will be dealt with in section 4.4. It remains to establish that the terms involving time derivatives do combine into the time derivative of a single positive definite quantity.

Clearly,

$$
\left(\Phi^{(\mathrm{c})}, \dot{\Phi}^{(\mathrm{c})}\right)_{I}=\frac{1}{2} \frac{d}{d t}\left(\Phi^{(\mathrm{c})}, \Phi^{(\mathrm{c})}\right)_{I} .
$$

Next,

$$
\left(K^{(\mathrm{c})}, \dot{\Phi}^{(\mathrm{c})}\right)_{I}=\sum_{i} K_{i}^{(\mathrm{c})} \dot{\phi}_{i}^{(\mathrm{c})}=\sum_{i} \sum_{e} \sum_{e^{\prime}} T_{i e e^{\prime}} V_{e} V_{e^{\prime}} \dot{\phi}_{i}^{(\mathrm{c})} .
$$

Finally,

$$
\begin{aligned}
(C, \dot{V})_{H}=\sum_{e} F_{e} \dot{V}_{e} & =\sum_{e} \sum_{e^{\prime}} H_{e e^{\prime}} \hat{\phi}_{e e^{\prime}} V_{e^{\prime}} \dot{V}_{e}=\frac{1}{2} \sum_{e} \sum_{e^{\prime}} H_{e e^{\prime}} \hat{\phi}_{e e^{\prime}} \frac{d}{d t}\left(V_{e^{\prime}} V_{e}\right) \\
& =\frac{d}{d t}(\ldots)-\frac{1}{2} \sum_{e} \sum_{e^{\prime}} H_{e e^{\prime}} V_{e^{\prime}} V_{e} \frac{d}{d t} \hat{\phi}_{e e^{\prime}} .
\end{aligned}
$$

Thus, the time derivative terms will combine into the time derivative of a single positive definite quantity, provided

$$
H_{e e^{\prime}} \hat{\phi}_{e e^{\prime}}=\sum_{i} T_{i e e^{\prime}} \phi_{i}^{(\mathrm{c})} .
$$

In other words, the $\phi$ values used to construct the mass flux must be built using the same weights $T_{i e e^{\prime}}$ as are used to construct the kinetic energy per unit mass. For any fixed $i, T_{i e e^{\prime}}$ must be symmetric in the indices $\left\{e, e^{\prime}\right\}$ and positive definite.

4.4. Energy conserving Coriolis term. An exactly energy conserving potential vorticity flux can be constructed using the method of [21]. From the primal vertex values of potential vorticity $q_{v}^{(\mathrm{v})}$, construct edge values of potential vorticity $\hat{q}_{e}$ using any desired interpolation scheme. Then define

$$
Q_{e}^{\perp}=\sum_{e^{\prime}}\left(\frac{\hat{q}_{e}+\hat{q}_{e^{\prime}}}{2}\right) W_{e e^{\prime}} F_{e^{\prime}}
$$

The symmetry of the two $\hat{q}$ terms combined with the antisymmetry of $W$ ensures that $\left(C, Q^{\perp}\right)_{H}=\sum_{e} F_{e} Q_{e}^{\perp}=0$.

An alternative approach, which may have practical benefits, is to define $Q^{\perp}=$ $\hat{q}_{e} C_{e}^{\perp}$, where

$$
C_{e}^{\perp}=\sum_{e^{\prime}} W_{e e^{\prime}} F_{e}
$$

is the mass flux that appears in the dual cell mass equation, and $\hat{q}_{e}$ is defined using an accurate advection scheme for the potential vorticity. This approach gives up exact energy conservation in exchange for an accurate treatment of potential vorticity. 
4.5. Choice of predicted velocity variable. For the linearized equations we could have chosen to predict $U$ rather than $V$ - simply apply $H$ to (2.10) to obtain the equivalent prognostic equation for $U_{e}$ - and all the desirable properties are retained. For the nonlinear equations, however, there is a clear preference for predicting $V$. If we predict $U$, then we need the operator $H^{-1}$ to construct the potential vorticity $q^{(\mathrm{v})}$ and the kinetic energy per unit mass $k^{(\mathrm{c})}$, as well as the operator $H$. At least one of $H$ or $H^{-1}$ will be a full matrix, making the scheme expensive, unless both are diagonal. If we predict $V$, on the other hand, we only need the operator $H$ even in the nonlinear case.

5. Discussion: Constraints on $I, J, H, R$, and $W$, and higher-order accuracy. The discrete derivatives $\bar{D}_{1}, D_{1}, D_{2}, \bar{D}_{2}$ are all exact (section 2.2). Therefore, the only approximations in the proposed framework are in the mapping operators $I, J, H, R$, and $W$. Thus, it may be possible to use the remaining freedom in the definitions of these operators to obtain higher-order accurate mimetic discretizations if higher-order operators can be constructed with the required symmetry properties. Note, however, that attainment of the mimetic properties does not, on its own, even guarantee consistency of the scheme.

A diagonal version of $I$,

$$
I_{i i}=1 / A_{i}^{(\mathrm{c})}
$$

where $A_{i}^{(\mathrm{c})}$ is the area of primal cell $i$, will be first-order accurate, provided the dual vertex $i$ lies within primal cell $i$. However, on a centroidal grid (see, e.g., [9]) the diagonal $I$ will be second-order accurate. A diagonal version of $H$,

$$
H_{e e}=l_{e} / d_{e}
$$

where $l_{e}$ is the length of primal edge $e$ and $d_{e}$ is the length of dual edge $e$, will in general be inconsistent (zeroth-order accurate) on a nonorthogonal grid because $\mathbf{m}_{e}$ and $\mathbf{n}_{e}$ point in different directions. On an orthogonal grid this diagonal $H$ will be first-order accurate, provided the primal and dual edges do intersect each other, and will be second-order accurate if the grid is constructed so that the intersection point approaches the midpoint of both the primal edge and the dual edge as resolution is increased [12].

More generally, a simple argument based on counting degrees of freedom suggests that constructing symmetric $I, J$, and $H$ operators of any desired order of accuracy may be possible. However, initial investigation of the one-dimensional problem for constructing $I$ suggests that the symmetry requirement is a significant constraint, and that higher-order accuracy may require the grid to have special properties, for example, that the dual vertices should approach the primal cell midpoints as resolution is refined.

The construction of a suitable higher-order $R$ operator also appears challenging. Provided $R$ satisfies (2.17), then it will be first-order accurate. If, in addition,

$$
\sum_{i \in \mathrm{CV}(v)} R_{v i} A_{i}^{(\mathrm{c})} \overline{\mathbf{x}}_{i}^{(\mathrm{c})}=A_{v}^{(\mathrm{v})} \overline{\mathbf{x}}_{v}^{(\mathrm{v})}
$$

where $\overline{\mathbf{x}}_{i}^{(\mathrm{c})}$ and $\overline{\mathbf{x}}_{v}^{(\mathrm{v})}$ are the centers of mass of primal cell $i$ and dual cell $v$, respectively, then $R$ will be second-order accurate. However, it is nontrivial to satisfy all three constraints (2.16), (2.17), and (5.3) simultaneously, and there may be insufficient 
degrees of freedom to do so on some grid structures if the stencil for $R$ is restricted to $\mathrm{CV}(v)$. Moreover, the construction of the weights $W$ from the weights $R$ has only been worked out for the case in which the stencil is $\operatorname{CV}(v)[29]$, and it is not clear whether it can be extended to larger stencils.

The construction of suitable higher-order $I, J$, and $H$ operators, with stencils as compact and homogeneous as possible, and the question of whether $W$ can be constructed for $R$ with a larger stencil, are the subject of ongoing investigation.

6. Relation to discrete exterior calculus. Discrete exterior calculus $[13,14$, $4,8]$ provides a powerful framework both for theoretical understanding and for practical development of numerical schemes that mimic the properties of the continuous exterior calculus (see, e.g., [5]). In exterior calculus notation, the shallow-water equations may be written

$$
\begin{aligned}
\star \dot{\phi}+d(\phi \tilde{u}) & =0, \\
\dot{\tilde{v}}+\zeta \star \tilde{v}+d(\phi+k) & =0,
\end{aligned}
$$

where $\phi$ is the geopotential 0 -form and $\star \phi$ is the corresponding 2 -form, $\tilde{v}=\tilde{g} \mathbf{v}$ is the circulation 1-form ( $\tilde{g}$ is the metric), $\tilde{u}=\star \tilde{v}$ is the volume flux 1 -form, $\zeta=f+\xi$, where $\star \xi=d \tilde{v}$ is the relative vorticity 2 -form, $\star k=(\tilde{v} \wedge \star \tilde{v}) / 2=(\tilde{v} \wedge \tilde{u}) / 2$ is the kinetic energy 2 -form, $d$ is the exterior derivative, and $\star$ is the Hodge star operator, which reversibly converts $r$-forms into $(n-r)$-forms in $n$ dimensions.

The discretization described in sections $2-4$ has a systematic correspondence to the exterior calculus form of the governing equations. $r$-forms are discretized as quantities integrated over $r$-dimensional elements of the grid $[4,8]$. The discrete exterior derivative is then given by a series of simple expressions $(2.5)-(2.8)$ that are exact when applied to discrete $r$-forms: this is the "commuting diagram property" $[13,4]$. Table 6.1 summarizes the correspondences between continuous quantities and operators and their discrete analogues.

The desirable properties listed in section 1.1 are obtained by the discrete system mimicking the continuous one in key ways. A C-grid discretization is obtained by ensuring that the predicted variables $\Phi^{(\mathrm{c})}$ and $V$ live on the top row in Figure 3.1. Mass conservation is obtained by predicting the geopotential 2-form and because the operator $D_{2}$ is exact. The curl of the pressure gradient vanishes because the discrete exterior derivative satisfies $\bar{D}_{2} \bar{D}_{1} \equiv 0$, mimicking the continuous $d d \equiv 0$.

The discretization includes discrete analogues of the $\star$ operator, namely, $I, J, H$, $I^{-1}, J^{-1}$, and $-H^{-1}$, allowing the construction of inner products (3.6), (3.7) that give discrete analogues of $(\tilde{\alpha}, \widetilde{\beta})=\int \tilde{\alpha} \wedge \star \tilde{\beta}$. The fact that $(\star)^{-1}=(-1)^{r(n-r)} \star$ for $\star$ acting on $r$-forms in $n$ dimensions explains the minus sign that appears in front of $H^{-1}$. One slightly counterintuitive feature of this correspondence is that, because of the "twist" introduced by the $\star$ operator, the volume flux across a primal edge $U$ corresponds to the tangential component of the volume flux 1-form $\tilde{u}=\star \tilde{v}$.

Energy conservation by the geopotential terms is obtained because $\bar{D}_{1}$ is minus the adjoint of $I D_{2} H$; this is a discrete analogue of the continuous property that $d$ is minus the adjoint of $\star d \star$. The other discrete analogues of this relation are listed in Table 6.1. In the nonlinear case we also require that the mapping from edges to primal cells defined by $T$ should be the adjoint of the mapping from dual vertices to edges so that a well-defined discrete kinetic energy density $\phi k$ can be constructed from the predicted variables.

Because of the presence of Coriolis terms in the governing equations, we cannot avoid introducing the discrete quantities in the bottom row of Figure 3.1. Although 
TABLE 6.1

Continuous quantities and operators and their discrete analogues.

\begin{tabular}{|l|l|l|}
\hline $\begin{array}{l}\text { Continuous variable } \\
\text { or operator }\end{array}$ & Discrete analogue & Location \\
\hline$\phi$ & $\phi^{(\mathrm{c})}$ & \\
$\tilde{v}$ & $\Phi^{(\mathrm{c})}$ & Dual vertex \\
$\Phi^{(\mathrm{v})}$ & Primal cell \\
$\tilde{u}=\star \tilde{v}$ & $V$ & Dual cell \\
$d \tilde{u}$ & $U$ & Dual edge \\
& $V^{\perp}$ & Primal edge \\
$\star \xi=\star(\zeta-f)$ & $\Delta^{(\mathrm{c})}$ & Dual edge \\
\hline$\star$ & $\Delta^{(\mathrm{v})}$ & Primal cell \\
& $\Xi^{(\mathrm{v})}$ & Dual cell \\
& $I$ & Dual cell \\
\hline \multirow{4}{*}{ id } & $I^{-1}$ & Primal cell to primal vertex \\
& $J$ & Primal vertex to primal cell \\
& $J^{-1}$ & Dual cell to dual vertex \\
& $H^{-1}$ & Dual vertex to dual cell \\
& $-H^{-1}$ & Dual edge to primal edge \\
& $\bar{D}_{1}$ & Primal edge to dual edge \\
& $D_{1}$ & Dual vertex to dual edge \\
& $D_{2}$ & Primal vertex to primal edge \\
& $\bar{D}_{2}$ & Primal edge to primal cell \\
& $-I D_{2} H$ & Dual edge to dual cell \\
& $J^{*}=-\star d \star$ & Dual edge to dual vertex \\
& $H^{-1} D_{1} J$ & Primal edge to primal vertex \\
& $-H \bar{D}_{1} I$ & Dual cell to dual edge \\
& $R$ & Primal cell to primal edge \\
& $W$ & Primal cell to dual cell \\
& $T$ & Primal edge to dual edge \\
& & Dual edges to and from primal cell \\
\hline
\end{tabular}

the averaging in going from the top row to the bottom row, with some attendant loss of accuracy, is unavoidable on a C-grid, we can at least ensure that it meets certain constraints. The discrete averaging operators $R$ and $W$ convert discrete 2forms from primal to dual cells and 1-forms from primal to dual edges, respectively. Their continuous analogues are simply the identity operator. Antisymmetry of the matrix $W$ ensures a discrete analogue of $(\tilde{v}, \star \tilde{v}) \equiv 0$ so that the Coriolis terms are energy conserving. The property $-\bar{D}_{2} W=R D_{2}$ ensures that the dual grid analogue of $d$ is compatible with the primal grid analogue of $d$ in the sense required to give stationary geostrophic modes in the linear constant $f$ case.

The various expressions for scalar and vector Laplacians given in section 3.6 may all be succinctly written

$$
\nabla^{2}=-\left(D D^{*}+D^{*} D\right)
$$

in direct analogy with the continuous exterior calculus expression

$$
\nabla^{2}=-\left(d d^{*}+d^{*} d\right)
$$

where $d^{*}=(-1)^{n(r-1)+1} \star d \star$ and $D^{*}=(-1)^{n(r-1)+1} \star D \star$ are, respectively, the continuous and discrete adjoints of the exterior derivative acting on $r$-forms in $n$ di- 
mensions, and $D$ and $D^{*}$ are shorthand for whichever of the discrete operators is applicable. One consequence of having a discrete analogue of the adjoint relation between $d$ and $d^{*}$ is that the proof of the discrete Helmholtz decomposition (section 3.7) then becomes very straightforward [8].

Bochev and Hyman [4] discuss the challenges of constructing a discrete Hodge star operator that is compatible with the discrete inner product and with the discrete adjoint of the exterior derivative. They observe that "... if a discrete $\star$ operation is required, then it must be made the primary object of the discrete framework and then used to define all other necessary structures." That is, in effect, what we have done here. Hiptmair [13] notes that the construction of a discrete $\star$ is greatly facilitated if the discrete $\star$ operator transfers forms between a primary and a secondary mesh, and that the matrix representation of $\star$ simplifies if the secondary mesh is a dual of the primary mesh; the corresponding matrix must be symmetric and positive definite. Again, these are properties of the scheme described here. On an orthogonal grid, diagonal discrete Hodge stars are possible; this is what is done in the TRiSK [29, 21] scheme. However, on a nonorthogonal grid we must give up the diagonal property of $H$; see section 5 .

7. Conclusions. We have shown that the TRiSK spatial discretization of the rotating shallow-water equations, which gives a number of desirable properties, can be extended to apply on nonorthogonal grids. TRiSK is a special case of the extended scheme that uses diagonal $I$ and $H$ operators. The extension also opens up the possibility of obtaining higher-order accuracy (though finding higher-order versions of the $R$ and $W$ operators appears to be very challenging).

The proposed discretization fits within the framework of discrete exterior calculus and exploits that framework to obtain several of its good properties. We have extended that framework to include the mapping operators from the primal to the dual grid that are needed to represent the Coriolis term and to obtain the desirable properties 5 and 6 associated with the treatment of that term.

Only a subset of the operators defined here are actually needed for a numerical solution of the shallow-water equations, namely, the discrete derivatives $\bar{D}_{1}, D_{2}, \bar{D}_{2}$ and the mapping operators $I, H, W$, and $T$ ( $R$ is contained implicitly in the construction of $W$ ). In order to obtain the desired properties of the scheme, the following must hold: (i) $I$ and $H$ must be symmetric and positive definite; (ii) for any fixed $i$, $T_{i e e^{\prime}}$ must be symmetric in $\left\{e, e^{\prime}\right\}$ and positive definite; (iii) $R$ must satisfy (2.16).

There is freedom to choose larger stencils for $I$ and $H$, and it may be possible to use this freedom to increase the order of accuracy of these operators. However, it is currently not known whether the stencil for $R$ can be extended beyond $\operatorname{CV}(v)$ while still being able to construct an antisymmetric $W$ such that $-\bar{D}_{2} W=R D_{2}$.

Although in Figure 2.1 we have depicted the grid as built of straight primal and dual edges, this is not a requirement of the scheme. The edges may be piecewise straight, or curved; provided we define the unit vectors $\mathbf{n}, \mathbf{t}, \mathbf{m}, \mathbf{s}$ to vary in the appropriate way along edges, the discretization remains valid. This may have some advantages in certain coordinate systems or domain geometries, though it may complicate the construction of accurate $I, H, R$, and $T$ operators.

Finally, note that, although the proposed scheme has great flexibility and several desirable properties, it does not avoid the need for averaging in computing the Coriolis term; this is an inherent property of the C-grid. Nor can it prevent the existence of computational modes; these arise because of the relative numbers of degrees of freedom in the predicted variables determined by the grid structure and staggering. 
Acknowledgments. The authors thank Hilary Weller, Nigel Wood, and two anonymous reviewers for valuable comments on earlier versions of this paper.

\section{REFERENCES}

[1] A. Arakawa And V. R. Lamb, Computational design of the basic dynamical processes of the UCLA general circulation model, Methods Comput. Phys., 17 (1977), pp. 174-265.

[2] A. Arakawa AND V. R. Lamb, A potential enstrophy and energy conserving scheme for the shallow-water equations, Mon. Weather Rev., 109 (1981), pp. 18-36.

[3] D. ARnold, R. FALK, AND R. Winter, Finite element exterior calculus, homological techniques and applications, Acta Numer., 15 (2006), pp. 1-155.

[4] P. B. Bochev and J. M. Hyman, Principles of mimetic discretizations of differential operators, Math. Appl., 142 (2006), pp. 89-120.

[5] W. L. Burke, Applied Differential Geometry, Cambridge University Press, Cambridge, UK, 1985.

[6] C. J. Cotter, D. A. Ham, And C. C. Pain, A mixed discontinuous/continuous finite element pair for shallow-water ocean modelling, Ocean Modelling, 26 (2009), pp. 86-90.

[7] S. DANILOV, On the utility of triangular C-grid type discretization for numerical modeling of large-scale ocean flows, Ocean Dynamics, 60 (2010), pp. 1361-1369.

[8] M. Desbrun, E. Kanso, And Y. Tong, Discrete differential forms for computational modeling, in Discrete Differential Geometry: An Applied Introduction, M. Desbrun, E. Grinspun, P. Schröder, and M. Wardetzky eds., SIGGRAPH Asia 2008 Course Notes, 2008, pp. 4662.

[9] Q. Du, V. Faber, And M. Gunzburger, Centroidal Voronoi tessellations: Applications and algorithms, SIAM Rev., 41 (1999), pp. 637-676.

[10] A. Gassmann And H.-J. Herzog, Towards a consistent numerical compressible non-hydrostatic model using generalized Hamiltonian tools, Quart. J. Roy. Meteorol. Soc., 134 (2008), pp. $1597-1613$.

[11] F. H. HaRlow And J. E. Welch, Numerical calculation of time-dependent viscous incompressible flow of fluid with free surface, Phys. Fluids, 12 (1965), pp. 2182-2189.

[12] R. Heikes AND D. A. RANDALL, Numerical integration of the shallow water equations on a twisted icosahedral grid. Part II: A detailed description of the grid and an analysis of numerical accuracy, Mon. Weather Rev., 123 (1995), pp. 1881-1887.

[13] R. Hiptmair, Discrete Hodge operators, Numer. Math., 90 (2001), pp. 265-289.

[14] A. N. Hirani, Discrete Exterior Calculus, Ph.D. Dissertation, California Institute of Technology, Pasadena, CA, 2003.

[15] B. J. Hoskins, M. E. McIntyre, And A. W. Robertson, On the use and significance of isentropic potential vorticity maps, Quart. J. Roy. Meteorol. Soc., 111 (1985), pp. 877946.

[16] J. M. Hyman and M. Shashrov, The orthogonal decomposition theorems for mimetic finite difference methods, SIAM J. Numer. Anal., 36 (1999), pp. 788-818.

[17] S. NičKović, M. Gavrilov, And I. TošIć, Geostrophic adjustment on hexagonal grids, Mon. Weather Rev., 22 (2002), pp. 668-683.

[18] R. A. Nicolaides, Direct discretization of planar div-curl problems, SIAM J. Numer. Anal., 29 (1992), pp. 32-56.

[19] M. Rančić, J. Purser, And F. Mesinger, A global shallow water model using an expanded spherical cube: Gnomonic versus conformal coordinates, Quart. J. Roy. Meteorol. Soc., 122 (1996), pp. 959-982.

[20] D. A. Randall, Geostrophic adjustment and the finite-difference shallow-water equations, Mon. Weather Rev., 122 (1994), pp. 1371-1377.

[21] T. D. Ringler, J. Thuburn, J. B. Klemp, and W. C. Skamarock, A unified approach to energy and potential vorticity dynamics for arbitrarily structured C-grids, J. Comput. Phys., 229 (2010), pp. 3065-3090.

[22] C. Ronchi, R. Iacono, And P. PaOlucci, The "cubed-sphere": A new method for the solution of partial differential equations in spherical geometry, J. Comput. Phys., 124 (1996), pp. 93-114.

[23] R. Salmon, A general method for conserving energy and potential enstrophy in shallow-water models, J. Atmos. Sci., 64 (2007), pp. 515-531.

[24] W. C. Skamarock, J. B. Klemp, M. G. Duda, L. Fowler, S.-H. Park, and T. D. Ringler, A multi-scale nonhydrostatic atmospheric model using centroidal Voronoi tessellations and C-grid staggering, Mon. Weather Rev., to appear (2012). 
[25] M. Sommer And P. NÉVIR, A conservative scheme for the shallow-water system on a staggered geodesic grid based on a Nambu representation, Quart. J. Roy. Meteorol. Soc., 135 (2009), pp. $485-494$.

[26] A. Staniforth and J. Thuburn, Horizontal grids for global weather and climate prediction models: A review, Quart. J. Roy. Meteorol. Soc., 138 (2012), pp. 1-26.

[27] J. Thuburn, Some conservation issues for the dynamical cores of NWP and climate models, J. Comput. Phys., 227 (2008), pp. 3715-3730.

[28] J. Thuburn, Numerical wave propagation on the hexagonal C-grid, J. Comput. Phys., 227 (2008), pp. 5836-5858.

[29] J. Thuburn, T. D. Ringler, W. C. Skamarock, and J. B. Klemp, Numerical representation of geostrophic modes on arbitrarily structured C-grids, J. Comput. Phys., 228 (2009), pp. 8321-8335.

[30] H. Weller, J. Thuburn, And C. J. Cotter, Computational modes and grid imprinting on five quasi-uniform spherical C-grids, Mon. Weather Rev., to appear (2012). 